\title{
TUNEABLE CURRENT MODE RMS DETECTOR
}

\author{
Predrag B. Petrović *
}

\begin{abstract}
A new realization of RMS detector, employing two CCCIIs (controlled current conveyors), metal-oxide-semiconductor transistors and single grounded capacitor is present in this paper, without any external resistors and components matching requirements. The proposed circuit can be applied in measuring the RMS value of periodic, band-limited signals. The proposed circuit is very appropriate to further develop into integrated circuits. The errors related to the signal processing and errors bound were investigated and provided. To verify the theoretical analysis, the circuit PSpice simulations have also been included, showing good agreement with the theory.
\end{abstract}

K e y w or d s: RMS detector, controlled current conveyors, MOSFET, multi-harmonic band-limited signals, simulation

\section{INTRODUCTION}

RMS (root-mean square) amplitude is a consistent, useful and standard way to measure and compare dynamic signals of all shapes and sizes. RMS detectors of various designs can be found in many applications in the fields of communication and of measurement systems $[1,2]$. RMS detectors based on Joule heating provide good accuracy and wide bandwidth. However, the rather complex packaging requirements do not lead to a low-cost solution. Diode detectors based on the square-law are traditionally employed in communication systems as a form of power measurement because of their favourable highfrequency performance and low-cost. However, elaborate compensation techniques are required to make them meet the demands of most applications. Dynamic range and temperature stability also limit their application.

Different methods have been reported for the precision measurement of the RMS value of an AC voltage, such as sampling [3], Monte Carlo [4], and the wavelet transform $[5,6]$. The implicit RMS converter described in [7-12], has been used for many years. Although many data sheets and textbooks include a mathematical discussion of the operation of these circuits, this type of RMS detector design is seen as insufficiently efficient in adequately processing the input multi-harmonic signals. Most of these devices are similarly built up from two main parts: a full-wave rectifier (or absolute-value) circuit and a multiplier/divider circuit employing a log-antilog principle. Due to the bandwidth and the slow rate of the full-wave rectifier, the highfrequency performances of these devices are limited to less than $5 \mathrm{MHz}$. Design techniques based on bipolar dynamic trans-linear circuits have been proposed to implement true RMS-to-DC converters $[13,14]$. Although these scheme require only NPN transistors, their circuits are operated in only one quadrant and employ full-wave rectifier. A new design technique for RMS-to-DC converter that design around a dual trans-linear-based squarer circuit is proposed in $[15,16]$, where the input current can be a two-quadrant current signal. Because the full-wave rectifier is not required by this conversion scheme, the circuit exhibits a wide bandwidth, but limited compared to thermal-based or diode-based detectors (due to input interference) [17].

Second-generation current conveyors (CCIIs), first introduced in [18], are functionally flexible and versatile. The current-mode circuits have been receiving considerable attention due to their potential advantages such as inherently wide bandwidth, higher slew-rate, greater linearity, wider dynamic range, simple circuitry and low power consumption. The CCII is a reported active component, especially suitable for a class of analogue signal processing. However, the CCII based circuit for measurement the RMS value of multi-harmonic voltage signal has not been reported so far [19]. On the other hand, the CCII can not control the parasitic resistance at $x$ $\left(R_{x}\right)$ port so when it is used in some circuits, it must unavoidably require some external passive components, especially the resistors. This makes it not appropriate for IC implementation due to occupying more chip area, high power dissipation and without electronic controllability. The introduced second-generation current controlled conveyor (CCCII) has the advantage of electronic adjustability over the CCII [20]. Also, the use of dual-output current-conveyors is found to be useful in the derivation of current-mode single input circuits [21-23].

The features of the proposed circuit are that: the circuit description is very simple; it employs two CCCIIs and single grounded capacitor as passive component, which is suitable for fabrication in monolithic chip, which makes it suitable for high-frequency operations [24]. The time constant of integrator can be electronically controlled [25]. Differently form previous works, exact integration on a period is performed instead of estimating the mean value

\footnotetext{
* Faculty of Technical Sciences Čačak, University of Kragujevac, Svetog Save 65, 32000 Čačak, Serbia, predrag.petrovic@ftn.kg.ac.rs
} 


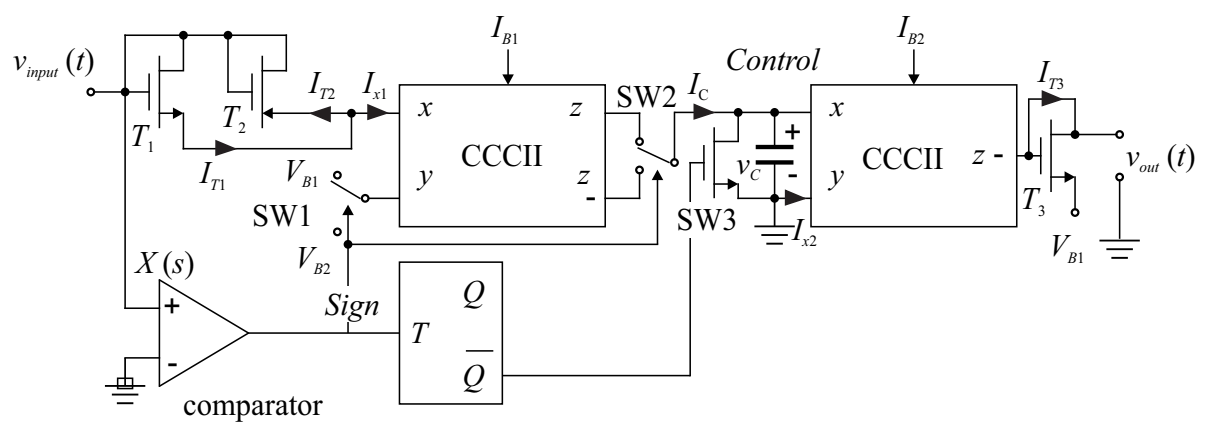

Fig. 1. The proposed realization of RMS circuit

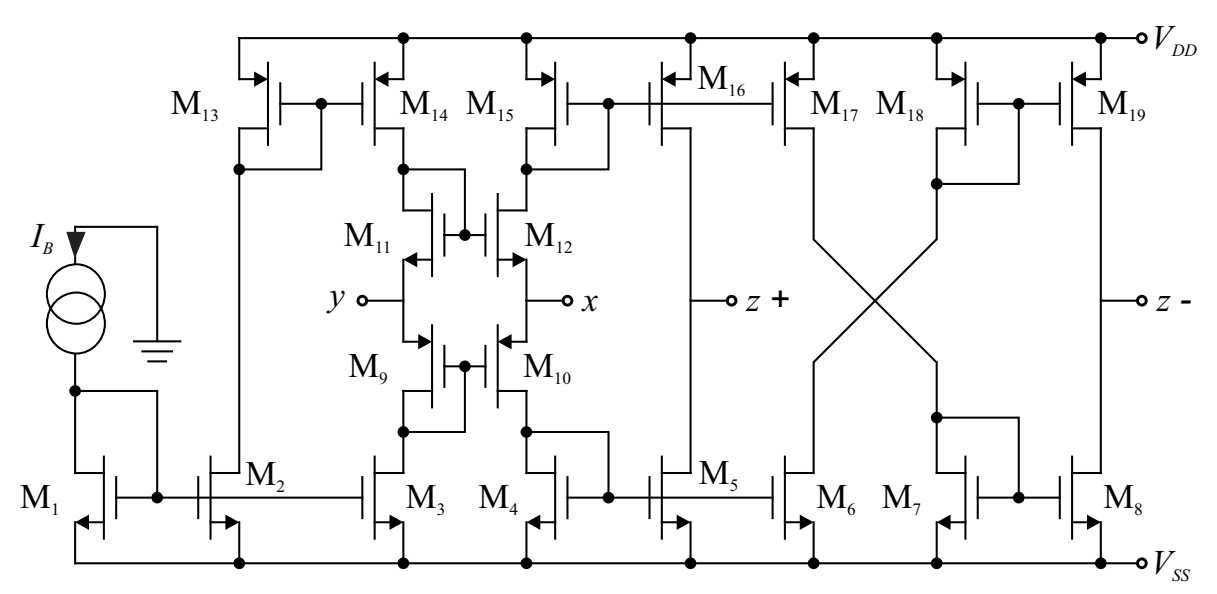

Fig. 2. Schematic of the CMOS CCCII

with a low pass filter. In terms of frequency range, the proposed circuit operation covers a wide range of as many as six decades, with increased linearity and precision in determining the effective value. The performance of the proposed circuit are illustrated by PSpice simulations, they show good agreement with the calculation.

\section{PROPOSED RMS MEASURING CIRCUIT}

The proposed circuit for measuring the RMS value of multi-harmonic, band-limited input signal is shown in Fig. 1. The NMOS transistors $\left(T_{1}, T_{3}\right)$ have positive threshold voltage $V_{T n}$, while the PMOS transistor $T_{2}$ has negative $V_{T p}$. The $y$ port of the first CCCII is biased at the threshold voltages of the MOS transistors as $V_{B 1}=$ $-V_{T n}$ and $V_{B 2}=-V_{T p}$.

By introducing self-biasing, it is possible to avoid the necessity for external biasing and the entailing requirements for special band gap bias circuits; since all the internal bias voltages and currents are generated from each other, the bias levels are completely determined by the operating conditions.

The characteristics of the ideal CCCII are represented by the following hybrid matrix

$$
\left[\begin{array}{c}
I_{y} \\
V_{x} \\
I_{z}
\end{array}\right]=\left[\begin{array}{ccc}
0 & 0 & 0 \\
1 & R_{x} & 0 \\
0 & \pm 1 & 0
\end{array}\right]\left[\begin{array}{c}
V_{y} \\
I_{x} \\
V_{z}
\end{array}\right] .
$$

If the CCCII is realized using CMOS technology, $R_{x}$ can be respectively written as

$$
R_{x}=\sqrt{\frac{1}{k I_{B}}} .
$$

Here $k$ is the physical trans-conductance parameter of the MOS transistor, defined as $k=8 \mu_{p} C_{\text {oxp }}\left(W_{p} / L_{p}\right)_{9-10}=$ $8 \mu_{n} C_{o x n}\left(W_{n} / L_{n}\right)_{11-12} . I_{B}$ is the bias current used to control the intrinsic resistance at $x$ port. In general, CCCII can contain an arbitrary number of $\mathrm{z}$ terminals; provide both directions of currents $I_{z}$. The internal construction of the CMOS CCCII is shown in Fig. 2.

It should be noted that the first CCCII in Fig. 1 should has low impendance $\left(R_{x 1} \cong 0\right)$ by setting value of $I_{B 1}$ at the highest possible value allowed by the proposed configuration of CCCIIs in order to achieve low input impedance of the first CCCII. The real limit of the bias current is defined after simulation checks in Section 4 . Also, the graph of the bias current vs $R_{x}$ will be also included. The second CCCII on Fig. 1 form current-mode inverting lossless integrator. Considering the circuit in Fig. 1 and using CCCII properties, the current transfer function of second CCCII is written as

$$
\frac{I_{T 3}}{I_{C}}=-\frac{1}{s C R_{x 2}} .
$$

If $v_{\text {input }}(t)>0$, the current is conducted through the NMOS transistors $T_{1}$ to the output. However, if 
Table 1. Uncertainty Budget (the size of the error in determining the RMS value of the input voltage, which occurs as a consequence of the non-ideal nature of the components applied in the circuit proposed in Figure 1)

\begin{tabular}{ccccccc}
\hline Parameter & Estimate $\begin{array}{c}\text { Standard } \\
\text { uncertainty } \\
(\%)\end{array}$ & Type Distribution & $\begin{array}{c}\text { Sensitivity } \\
\text { coefficient }\end{array}$ & $\begin{array}{c}\text { Contribution to } \\
\text { the standard } \\
\text { uncertainty (\%) }\end{array}$ \\
\hline$R_{x 2} C$ & $0.020 \mathrm{~ms}$ & 0.58 & $\mathrm{~B}$ & Uniform & -0.50 & 0.29 \\
$t_{1}$ & $0.020 \mathrm{~ms}$ & 0.58 & $\mathrm{~B}$ & Uniform & 3.00 & 1.73 \\
$\alpha_{i}$ & 1 & 0.58 & $\mathrm{~B}$ & Uniform & 0.50 & 0.29 \\
$\Delta V_{T}$ & 1 & 0.58 & $\mathrm{~B}$ & Uniform & 0.50 & 0.29 \\
$k_{p} / k_{n}$ & 0 & 0.58 & $\mathrm{~B}$ & Uniform & 0.50 & 0.29 \\
$\lambda_{i} v_{D S i}$ & 1 & $0.58^{*}$ & $\mathrm{~B}$ & Uniform & 0.50 & 0.29 \\
$e$ & 0 & & & & & 1.91 \\
\hline
\end{tabular}

$v_{\text {input }}(t)<0$, the PMOS transistor $T_{2}$ conducts the current to the output. Hence, the current-voltage relationships of the MOS transistors are given by

$$
\begin{aligned}
& I_{T 1}=\frac{k_{n}}{2}\left(v_{G S 1}-V_{T n}\right)^{2}=\frac{k_{n}}{2} v_{\text {input }}^{2}(t), \text { for } v_{\text {input }}(t)>0, \\
& I_{T 2}=\frac{k_{p}}{2} v_{\text {input }}^{2}(t), \text { for } v_{\text {input }}(t)<0 .
\end{aligned}
$$

NMOS and PMOS transistors conduct in opposite halves of the input signal. Depending on the detected sign of the input signal (the control signal Sign), over the comparator, the position of the switches SW1 and SW2 can be determined (CMOS analogue switch like single-pole double-throw Maxim MAX319), and the corresponding threshold voltage is connected to $y$ port of the CCCII. Such control enables current input from the port $z+$ on the integrator at the interval in which the input voltage signal is positive, ie from the port $z$ - when the input voltage is negative. If the input amplitude is below $V_{T n(p)}$ as in sub threshold circuits (very rare situation in practical applications), we will need to boost the level of the input signal. Assume that

$$
k_{n(T 1-T 3)}=k_{p(T 2)} \text {. }
$$

It follows that:

$$
I_{C}=-\left(I_{T 1}+I_{T 2}\right)=-\frac{k}{2} v_{\text {input }}^{2}(t) .
$$

The voltage formed in this way at the condenser $C$ will generate current which will be equalized with the current of the $T_{3}$ transistor

$$
I_{T 3}=\frac{1}{R_{x 2} C} \frac{k}{2} \int_{0}^{t_{1}} v_{\text {input }}^{2}(\tau) \mathrm{d} \tau=\frac{k}{2} V_{\text {out }}^{2}\left(t_{1}\right) .
$$

From the above, it follows that

$$
V_{\text {out }}\left(t_{1}\right)=\sqrt{\frac{1}{R_{x 2} C} \int_{0}^{t_{1}} v_{\text {input }}^{2}(\tau) \mathrm{d} \tau} .
$$

By choosing $R_{x 2} C=T$ and $t_{1}=T=1 / f$, where $T$ is the period of the input complex signal, it is clear that relation (8) represents a definition expression based on which it is possible to calculate the effective value of the input complex voltage signal. It is important to note that (8) is valid for any input signal (sine-, square, triangular-wave), which is known to have a $T$ period. The control signal Control can be generated in accordance with the detected zero-crossing of the input signal, over the edge-triggering $T$ flip-flop. The flip flop toggles at any negative-to-positive sign reversal. Integration over a period occurs only if there is only a single zero-crossing transition (with positive slope) in a signal period, which is valid for most real signals. In the period in which the SW3 close, the $C$ condenser is discharged to the zero voltage, thus preparing the integrator for the next round of charging process.

This kind of processing does not introduce limitations regarding the type of the signal and number of harmonics, that is being processed, ie this signal can contain nonharmonic components as well (inter-harmonics and subharmonics). However, it will be necessary to determine the period of such a complex signal, as it was done in [26]. The proposed circuit is very appropriate for hardware realization in integrated technology, and posses much simpler structure than circuits described in [7, 27-29].

\section{ERROR ANALYSIS}

Taking into account the non-ideal current gains of the CCCIIs $\alpha_{1}$ and $\alpha_{2}$ (ignoring the effects of voltage gains), the output voltage (Fig. 1) is given by

$$
\begin{aligned}
& V_{\text {out }}\left(t_{1}\right)=\sqrt{\frac{1}{R_{x 2} C} \int_{0}^{t_{1}}\left(\alpha_{1} v_{\text {input }}^{2}(\tau)_{+}+\alpha_{2} v_{\text {input }}^{2}(\tau)_{-}\right) \mathrm{d} \tau} \\
& v_{\text {input }}(t)_{+}= \begin{cases}v_{\text {input }}(t), & \text { for } v_{\text {input }}(t)>0 \\
0, & \text { otherwise }\end{cases} \\
& v_{\text {input }}(t)_{-}= \begin{cases}v_{\text {input }}(t), & \text { for } v_{\text {input }}(t)<0 \\
0, & \text { otherwise }\end{cases}
\end{aligned}
$$

Therefore, the parameters $\alpha_{1}$ and $\alpha_{2}$ are in the form of multiplier constants for cycles (two) of input voltage. 
If equation (5) is not satisfied, the following inequality is obtained

$$
\begin{aligned}
& V_{\text {out }}\left(t_{1}\right)= \\
& \sqrt{\frac{1}{R_{x 2} C} \int_{0}^{t_{1}}\left(\alpha_{1} v_{\text {input }}^{2}(\tau)_{+}+\alpha_{2} \frac{k_{p}}{k_{n}} v_{\text {input }}^{2}(\tau)_{-}\right) \mathrm{d} \tau} .
\end{aligned}
$$

It is observed in (10) that the square of the negative cycle of input signal is multiplied by $k_{p} / k_{n}$ instead of unity. Fortunately, using ECCIIs [30], $\alpha_{1}=\alpha_{2} k_{p} / k_{n}=1$ can be adjusted. It is obvious that the relation (8) is valid under the ideal condition: $R_{x 2} C=T=t_{1} ; \alpha_{1}=1$; $\alpha_{2}=1 ; k_{p} / k_{n}=1 ; \lambda_{i} v_{D S i}=0, i=[1,2,3]$, where $\lambda$, the channel-length modulation parameter, models current dependence on drain voltage due to the Early effect, or channel length modulation. In proposed circuits $v_{D S i}=$ $v_{G S i}$.

If the body-effect is considered, body-source voltage affects the threshold voltage, which is shown in equation

$$
V_{T}=V_{T 0}+\Delta V_{T}=V_{T 0}+\gamma\left(\sqrt{V_{S B}+2 \varphi_{F}}-\sqrt{2 \varphi_{F}}\right)
$$

where $V_{T}$ is the threshold voltage when substrate bias is presented, $V_{S B}$ is source-to-body substrate bias, $2 \varphi_{F}$ is surface potential, and $V_{T 0}$ is threshold voltage for zero substrate bias, $\gamma=\left(t_{o x} / \varepsilon_{o x}\right) \sqrt{2 q \varepsilon_{s i} N_{A}}$ is body effect parameter, $t_{o x}$ is oxide thickness, $\varepsilon_{o x}$ is oxide permittivity, $\varepsilon_{s i}$ is permittivity of silicon, $N_{A}$ is doping concentration and $q$ is charge of an electron. In the proposed peak detector, each body of every MOS transistor is connected to its source $\left(V_{S B}=0\right)$ then $V_{T}=V_{T 0}$, except for central positioned NMOS transistors in CCCIIs and transistors $T_{1}, T_{2}$, and $T_{3}$ (Fig. 1). The body of transistor $T_{3}$ is actually connected to $V_{S S}$, while its drain provides the voltage output. This output offset voltage cannot be eliminated. When a small deviation offset occurs, the output voltage, $v_{\text {out }}(t)$, in non-ideal condition becomes

$$
\begin{aligned}
& V_{\text {out }}\left(t_{1}\right)= \\
& \quad\left[\frac { 1 } { ( 1 + \lambda _ { 3 } v _ { D S 3 } ) R _ { x 2 } C } \int _ { 0 } ^ { t _ { 1 } } \left(\alpha_{1} v_{\text {input }}^{2}(\tau)_{+}\left(1+\lambda_{1} v_{D S 1}\right)\right.\right. \\
& \left.\left.\quad+\alpha_{2} \frac{k_{p}}{k_{n}} v_{\text {input }}^{2}(\tau)_{-}\left(1+\lambda_{2} v_{D S 2}\right)\right) \mathrm{d} \tau\right]^{1 / 2}+\Delta V_{T}
\end{aligned}
$$

A question is raised as of the nature of the output voltage in the proposed RMS detector in circumstances when ideal conditions are not met. For any divergence in the value of the parameter in relation to its nominal value, it is possible to determine the value of the output voltage and calculate the ensuing error. For example, in (12), parameter $\alpha_{3}$ is replaced with $\left(1+\delta \alpha_{3} / 100\right) \alpha_{3}$, where $\delta \alpha_{3}$ represents the percentage divergence in the value of the $\alpha_{3}$ parameter in relation to its nominal value, then the value of the output voltage $V_{\text {out }}$ is calculated. After this, the relative error in the measuring is calculated

$$
e=\frac{V_{\text {out }}-V_{\text {eff }}}{V_{\text {eff }}} 100 .
$$

But, how large is the uncertainty of error $e$, calculated in this way, if there are known the partial uncertainties of the parameters considered? The answer to this question ought to represent the uncertainty budget which is based on the procedures described in GUM [31], and has been shown in Table 1.

The values in Table 1 correspond to the case where all the parameters of interest are known within the limits of $\pm 1 \%$ in relation to their nominal values, based on a uniform distribution of probability. Further on, the input voltage is formed in such manner that its DC component, as well as amplitudes and phases of its harmonics, are set in random manner (uniform distribution), respectively. The specific nature of the uncertainty budget set in this manner is reflected in the fact that for certain parameters it is not possible to establish exact values for sensitivity coefficients, since the particular values are dependent on the form of the input voltage $v_{\text {input }}(t)$.

Intervals for possible values of those sensitivity coefficients, as well as their distributions, can be determined by fairly large number of simulation of the measuring procedure, by varying parameter values and harmonic content of input voltage. (*Sensitivity to changes in certain factors is a function of the form of the input voltage $v_{\text {input }}(t)$; ${ }^{*}$ for practical reasons, uncertainty of parameters $\lambda_{i} v_{D S i}$ has been expressed in percentages of one.).

- Sensitivity coefficients for parameters

$R_{x 2} C$ and $\lambda_{i} v_{D S i}$ do not depend on the waveform of input voltage and are known exactly.

- For parameters $\alpha_{i}, \lambda_{i} v_{D S i}$ and $k_{p} / k_{n}$, it was found that sensitivity coefficients have the values in the interval from 0.0 to 0.5 , with approximately uniform distributions.

- Sensitivity coefficient for parameter $t_{1}$ extends from 0.0 to approximately 3.0 , with approximately hyperbolic distribution.

In Table 1, sensitivity coefficients take their maximum values. For this case, the combined uncertainty is $1.9 \%$, consequently, the expanded uncertainty (for a coverage factor $k=2$ ) is $3.8 \%$. It is clear that this figures are overestimated, but, for which amount? The GUM procedures do not offer the answer.

Regardless of the fact whether the base for the analysis of the uncertainty will be the uncertainty budget given in Table 1, this Table is useful because it yields information on the extent at which the variations in certain parameters influence the precision of measuring.

If some entries in Table 1 already are determined using simulations, varying parameters values and waveform of input voltage, it is reasonable to attempts that complete treatment of uncertainty estimation would be based on simulation of measuring procedure, as it is suggested in [31]. It can be expected that this approach offer more realistic uncertainty evaluation, given the fact that it does not 
Table 2. Dimensions of the Transistors

\begin{tabular}{ccc}
\hline Transistor & $\mathrm{W}(\mu \mathrm{m})$ & $\mathrm{L}(\mu \mathrm{m})$ \\
\hline M1-M8 & 5 & 0.5 \\
M9-M10 & 16 & 0.25 \\
M11-M12 & 8 & 0.25 \\
M13-M15, M17-M19 & 15 & 0.5 \\
M16 & 15.1 & 0.5 \\
\hline
\end{tabular}

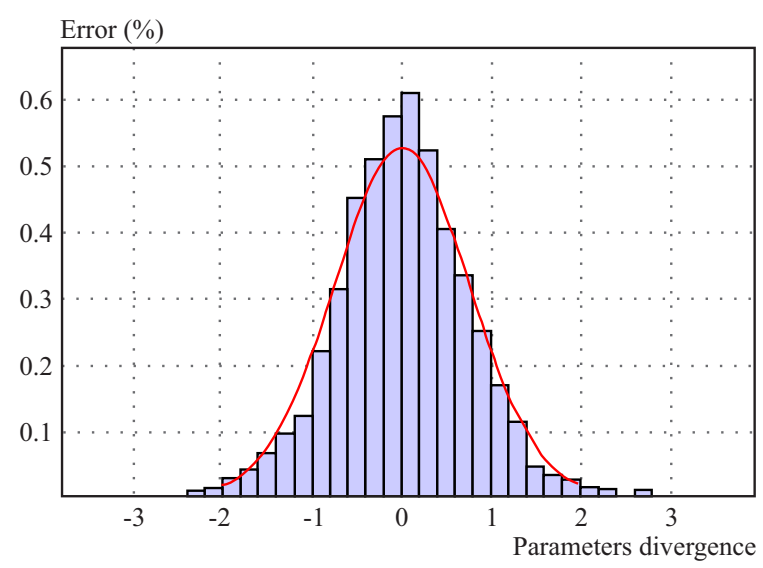

Fig. 3. The distribution of errors, for the divergence in the value of the parameters, from their nominal values

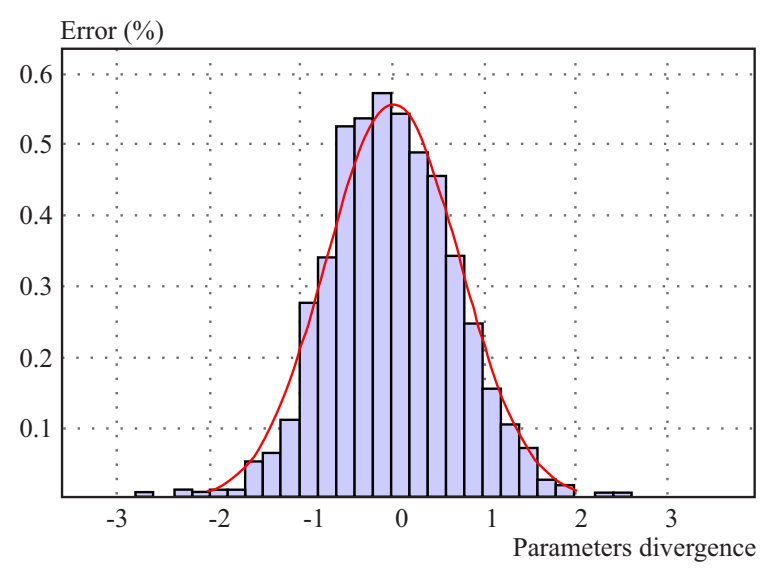

Fig. 4. The distribution of errors, for the divergence in the value of the parameters, from their nominal values in case of processing the signal contain inter-harmonics

imply any assumptions, neither regarding the distribution of the output value - the error in the measuring results, nor the distributions of the sensitivity coefficients values. Therefore, under the identical assumptions, we assumed that the input voltage has randomly set DC component, and amplitudes and phases of its harmonics; the observed parameters gain, in random manner, the value within the range of $\pm 1 \%$ around their nominal values. The result of implementation of the Monte Carlo variants is shown in Fig. 3 .

The expanded measuring uncertainty obtained here amounts to $1.5 \%$, and it ought to be compared with the data obtained from the uncertainty budget $(3.8 \%$, ie $1.6 \%)$. The estimated error is much lower then in case of RMS detector presented in [27]. The number of individual simulations in the presented case was 2200 . The simulations were performed assuming that the input voltage $v_{\text {input }}(t)$ has a DC component and ten harmonics, with randomly chosen amplitudes and phases. An almost identical result is obtained in the event when the selected number of harmonics is 3 .

\subsection{Impact of inter-harmonics and sub-harmonics}

The experiments were repeated, in case the input voltage $v_{\text {input }}(t)$ should contain inter-harmonics, in addition to harmonics. The input voltage is represented by

$$
\begin{aligned}
& v_{\text {input }}(t)=V_{0}+\sum_{i=1}^{n} V_{i} \sin \left(i \omega t+\varphi_{i}\right)+ \\
& \sum_{i=1}^{n-1} V_{i}^{\prime} \sin \left(\frac{i}{n} \omega t+\varphi_{i}^{\prime}\right)+\sum_{i=2}^{n-1} V_{i}^{\prime \prime} \sin \left(\frac{n}{i} \omega t+\varphi_{i}^{\prime \prime}\right) .
\end{aligned}
$$

For the reasons of simplification, ie alleviating the complexity of concrete calculations and reducing the time for performing simulations, it was decided to apply $n=3$. No suppositions were made concerning the amplitudes and phases of certain harmonics ( $i e$ the fact that amplitudes of harmonics in real systems tend to decrease, the later in the sequence they occur, etc.). Parameters $V_{0}, V_{i}^{\prime}, V_{i}^{\prime \prime}, \varphi_{i}, \varphi_{i}^{\prime}, \varphi_{i}^{\prime \prime}$ will gain random values within the range from 0 to 1 , ie within the range from 0 to $2 \pi$. The number of individual simulation was 1500 (Fig. 4). The measuring uncertainty obtained here amounts to $1.4 \%$. It can therefore be concluded that the system functions equally well when inter-harmonics are added to the input voltage.

\section{SIMULATION RESULTS}

The working of the proposed circuit has been verified using PSpice simulation program. The PMOS and NMOS transistors have been simulated by respectively using the parameters of a $0.25 \mu \mathrm{m}$ TSMC CMOS technology [32]. The comparator has been simulated on base of the realization described in [33]. Comparator detects the passing of the voltage signal through zero, this way achieving synchronization of the measuring cycle with the frequency of the processed signal. This comparator triggers at about $2.5 \mathrm{mV}$, so the error is about $20 \mathrm{~ns}$. This error can be ignored since there is no accumulation. Switches SW1-SW3 have been simulated using the parameters of CMOS analog switches MAX319/318 [34]. The positive-edge-triggered T flip-flop has been simulated using the parameters of D flip-flop 74LVC1G80 [35]. The aspect ratios of a PMOS and NMOS transistor are listed in Table 2. Figure 2 depicts schematic description of the CCCII used in the simulations. The circuit was biased with $\pm 1.25 \mathrm{~V}$ supply voltages, $C=0.1 \mathrm{nF}, I_{B 1}=260 \mu \mathrm{A}$ and $I_{B 2}=100 \mu \mathrm{A}$. In addition, $V_{B 1}=-0.4238 \mathrm{~V}$ and $V_{B 2}=0.5536 \mathrm{~V}$ are chosen. 


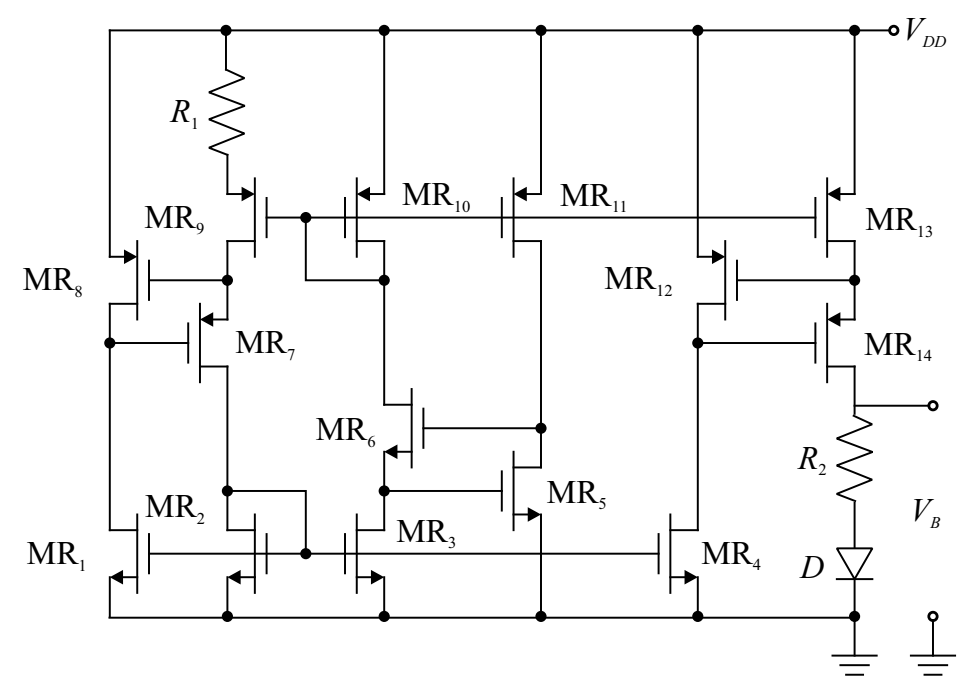

Fig. 5. CMOS voltage reference circuits

Table 3. Simulation results of proposed RMS circuit in the SPICE program

\begin{tabular}{cllll}
\hline Type of input signal & $\begin{array}{l}\text { Amplitude } \\
(\mathrm{V})\end{array}$ & $\begin{array}{l}\text { Phase } \\
(\mathrm{rad})\end{array}$ & $\begin{array}{c}\text { Obtained } \\
\text { RMS value }\end{array}$ & $\begin{array}{c}\text { Relative error } \\
\text { of measured } \\
\text { RMS value } \\
\text { of voltage (\%) }\end{array}$ \\
\hline & $\begin{array}{l}V_{1}=0.3 \\
V_{2}=0.25\end{array}$ & $\begin{array}{l}\psi_{1}=0 \\
\psi_{2}=\pi / 2\end{array}$ & & \\
Multi-harmonic sine signal & $\begin{array}{l}V_{3}=0 \\
V_{3}=0\end{array}$ & 0.291 & 0.13 \\
$v_{\text {input }}(t)=\sum_{r=0}^{N} V_{r} \sin \left(r \omega t+\psi_{r}\right)$ & $V_{4}=0.12$ & $\psi_{4}=\pi / 3$ & & \\
& $V_{5}=0.05$ & $\psi_{5}=\pi$ & & \\
Square-wave input signal & 0.5 & & 0.499 & 0.16 \\
Triangular-wave input signal & 0.5 & & 0.288 & 0.14 \\
\hline
\end{tabular}

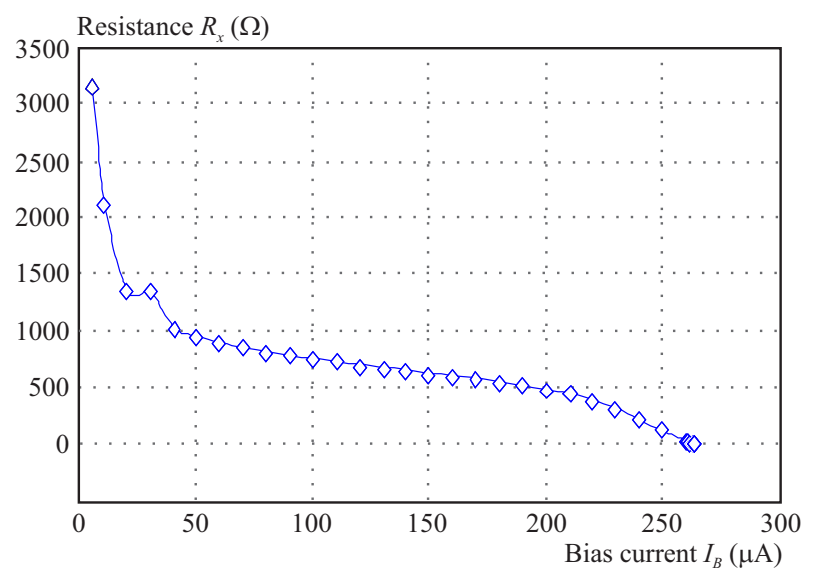

Fig. 6. Dependency of the port resistance of the bias current of the proposed CCCII

The possible design of such bias voltages is shown in Fig. 5, based on circuits proposed in [36]. In contrast to the circuit described in [36], the simulation check involved the parameters defined in [32], at temperatures $T=-45^{\circ} \mathrm{C}, T=25^{\circ} \mathrm{C}$ and $T=145^{\circ} \mathrm{C}$. At room temperature, for supply voltage of $1.25 \mathrm{~V}$ circuits generate voltage $V_{B}=0.424 \mathrm{~V}$ operating at $1.7 \mu \mathrm{A}$ supply current, and with $\sigma=0.81 \%$ (reference voltage 1 sigma spread). Within the observed temperature range (from $-45^{\circ} \mathrm{C}$ to $+145^{\circ} \mathrm{C}$ ), the temperature coefficient $T C$ of the proposed circuits was $30 \mathrm{ppm} / \mathrm{Cels}$. The circuit specified in [37] can also be used along with the solution described in the paper.

Figure 6 shows the relation of the bias current $I_{B}$ vs port resistance $R_{x}$ for the proposed realization of the CCCII, implying the real limits of the bias currents in the possible practical implementation of the proposed RMS detector. To achieve the conditions defined in Section 2, the bias current needs to be set as $I_{B 1}=260 \mu \mathrm{A}$ so as to obtain low input port resistances of the first CCCII. By changing the bias current $I_{B 2}$ (second CCCII) we can change the time constant of the realized integrator.

During the simulation, the parameters of the input signals correspond to the values given in Table 3 , with fundamental frequency $f=1 / T=2.5 \mathrm{MHz}$. Figures 7 and 8 shows the typical performance characteristics of the proposed detector.

The results shown in Table 3 and on Figs. 7 and 8 confirm the possibility of highly-precise determination of the effective value of the input multi-harmonic signal, by using the proposed circuit. An error in calculating the RMS value of multi-harmonic signals is smaller than the 

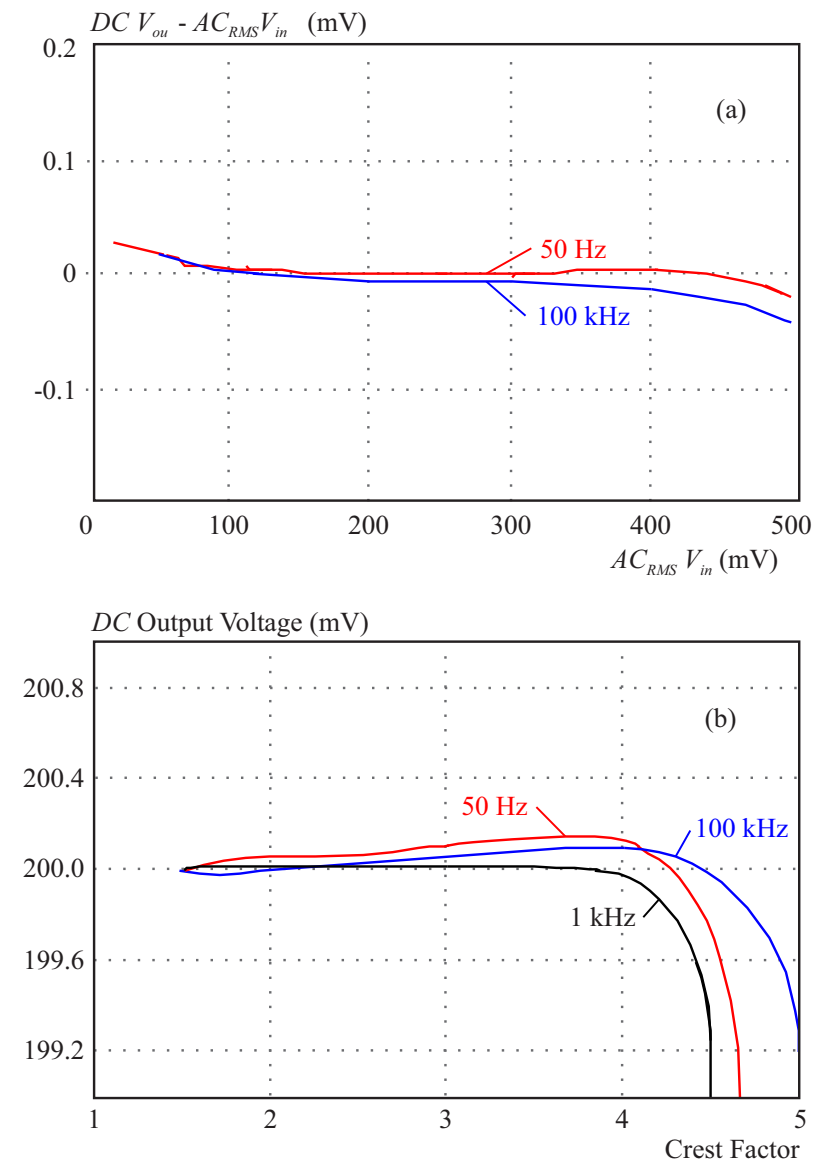

Fig. 7. (a) - Performance $v s$ crest factor, b - AC linearity

detector proposed in $[10,38,39]$, where the structure of the proposed circuits are much simpler then in [27].

In many applications, particularly in sensor signal processing, the inputs and outputs need to be analogue so that the unlimited resolution and accuracy that is possible with purely digital circuitry will not be available [40].

\section{CONCLUSION}

In this paper an electronically tuneable current-mode RMS detector for measuring the RMS value of multiharmonic, band-limited input voltage signal has been presented. The proposed configuration is simple and can be electronically controlled. The proposed circuit require only single grounded capacitor as passive element, which is advantageous in integrated circuit implementation and high-frequency operations point of view. The calculation of the effective value has been performed in full accordance with the definition formula, successfully overcoming almost all of the shortcomings that hindered the calculation of the effective value using the realizations known so far. The PSpice simulation results were depicted, and agree well with the theoretical anticipation.
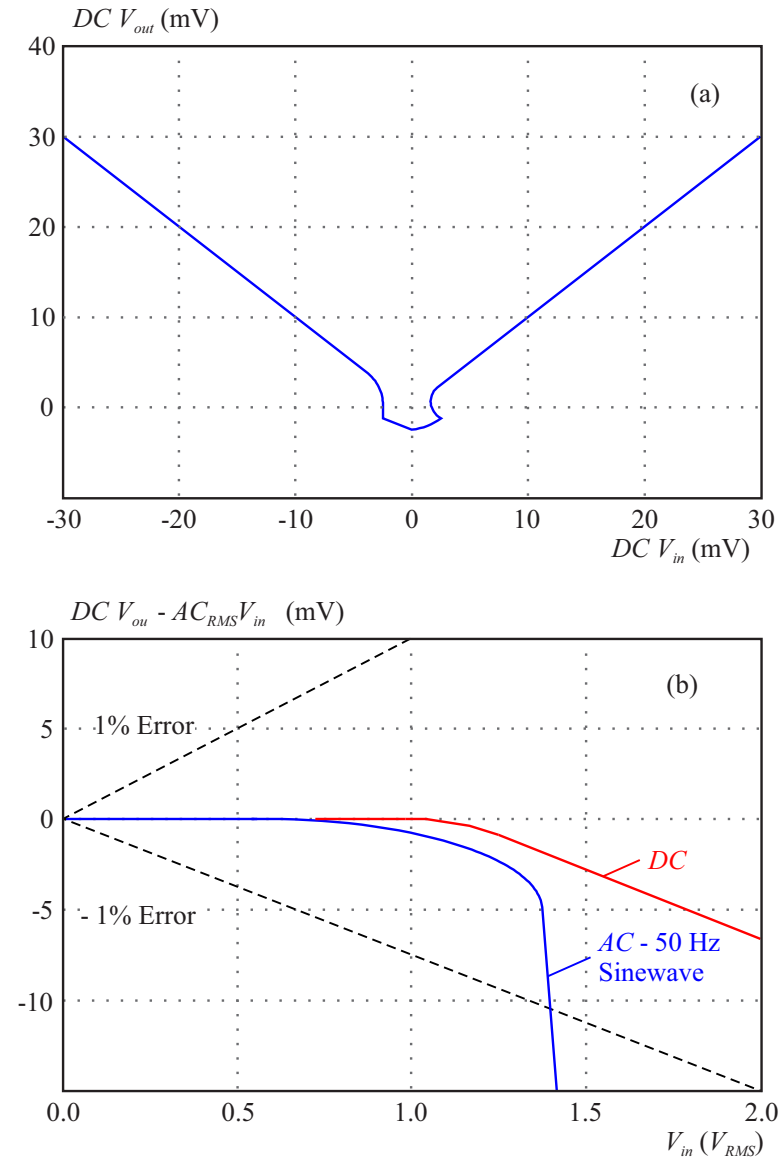

Fig. 8. (a) - DC transfer function near zero, (b) - output accuracy vs signal amplitude

\section{Acknowledgements}

The author wishes to thank to the Ministry of Education and Science of the Republic of Serbia for its support of this work provided within the projects 42009 and OI172057.

\section{REFERENCES}

[1] NORThrop, R. B.: Analog Electronics Circuits, AddisonWesley, Reading, MA, 1990.

[2] HeAvey, P.-Whitney, C.: RMS Measuring Principles in the Application of Protective Relaying and Metering, in Proc. 57th Annu. Conf. Protective Relay Eng., 2004, pp. 469-489.

[3] POGLIANA, U.: Precision Measurement of ac Voltage Below $20 \mathrm{~Hz}$ at IEN, IEEE Trans. Instrum. Meas. 46 No. 2 (1997), $369-372$.

[4] GERMER, H.: High-Precision AC Measurements using the Monte-Carlo Method, IEEE Trans. Instrum. Meas. 50 No. 2 (2001), 457-460.

[5] YOON, W. K.-DEVEnEY, M. J.: Power Measurement using the Wavelet Transform, IEEE Trans. Instrum. Meas. 47 No. 5 (1998), 1205-1210.

[6] NOVOTNY, M.-SEDLACEK, M.: RMS Value Measurement based on Classical and Modified Digital Signal Processing Algorithms, Measurement 41 No. 3 (2008), 236-250.

[7] True RMS' detector, National Semiconductor Application Note AN008474, 2002.

[8] DSCA33 ISOLATED True RMS Input Module, 2001, AN101 Dataforth Corporation, USA. 
[9] FREY, D. R.: Exact Analysis of Implicit RMS Converters, Electronics Letters 40 No. 5 (2004), 283-284.

[10] ABULMA'ATTI, M. T. : Improved Analysis of Implicit RMS Detectors, IEEE Trans. Instrum. Meas. 58 No.3 (2009), 502-505.

[11] High Precision, Wide-Band RMS-to-DC Converter, Analog Devices Application Note AD637, 2004.

[12] Precision Wide Bandwidth, RMS-to-DC Converter, Linear Technology Application Note LTC1968, 2008.

[13] MULdER, J.-SERDiJN, W. A.-WOERD, A. C.-ROERMUND, A. H. M. : Dynamic Translinear RMS-DC Converter, Electron Lett. 32 (1996), 2067-2068.

[14] MULDER, J.-SERdiJn, W. A.-ROERMUND, A. H. M. : An RMS-DC Converter Base don the Dynamic Translinear Principle, IEEE Solid-State Circuits 32 (1997), 1146-1150.

[15] SURAKAMPONTRON, W.-KUMWACHARA, K.: A Dual Translinear-Based RMS-to-DC Converter, IEEE Trans. Instrum. Meas. 47 (1999), 456-464.

[16] WASSENEAAR, R. F.-SEEVINCK, E.-van LEEUWEN, M. G.-SPEELMAN, C. J.-HOLLE, E.: New Techniques for High-Frequency RMS-to-DC Conversion Based on a Multifunctional V-to-I Convertor, IEEE Jour. Sol. Sta. Circ. 23 No. 3 (1998), 802-815.

[17] MilanoviĆ, V.-Gaitan, M.-BOWEN, E. D.-TEA, N. H.-ZAGHLOU, M. E. : Thermoelectric Power Sensors for Microwave Applications by Commercial CMOS Fabrication, IEEE Elec. Dev. Lett. 18 No. 9 (1997), 450-452.

[18] SEDRA, A. S.-SMITH, K. C.: A Second-Generation Current Conveyor and its Applications, IEEE Trans. Circuit Theory CT-17 No. 1 (1970), 132-134.

[19] YUCE, E.-MINAEI, S.-TOKAT, S.: Root-Mean-Square Measurement of Distinct Voltage Signals, IEEE Trans. Instrum. Meas. 56 No. 6 (2007), 2782-2787.

[20] FABRE, A.-SAAID, O.-WIEST, F.-BOUCHERON, C. : Current Controllable Bandpass Filter based on Translinear Conveyors, Electron. Lett. 31 (1995), 1727-1728.

[21] PAL, K.: Modified Current Conveyors and their Applications, Microelectronics Journal 20 (1989), 37-40.

[22] SOLIMAN, A. M. : Modified Current Conveyor Filters: Classification and Review, Microelectronics Journal 29 (1998), 133-149.

[23] TANGSRIRAT, W.-SURAKAMPONTORN, W. : High Output Impendance Current-Mode Universal Filter Employing Dual-Output Current-Controlled Conveyors and Grounded Capacitors, AEU-International Journal of Electronics and Communications 61 (2007), 127-131.

[24] FABRE, A.-SAAID, O.-BARTHELEMY, H. : On the Frequency Limitations of the Circuits based on the Second Generation Current Conveyors, Analog Integr. Circuits Signal Process. 7 No. 2 (1995), 113-129.

[25] PETROVIĆ, P. : Root-Mean-Square Measurement of Periodic, Band-Limited Signals, in Proc. of 2012 IEEE International Conference on Instrumentation and Measurement Technology (I2MTC), 2012, pp. 323-327.

[26] Petrovic, P.-Stevanovic, M.: IEE Proc. Elec. Pow. Appl. 153 No. 2 (2006), 227-235.

[27] ZHANG, T.-EISENSTADT, W. R.-FOX, R. M.-YIN, Q. : Bipolar RMS Power Detectors, IEEE Jour. Sol. Sta. Circ. 41 No. 9 (2006), 2188-2192.
[28] RUMBERG, B.-GRAHAM, D. W. : A Low-Power Magnitude Detector for Analysis of Transient-Rich Signals, IEEE Jour. Sol. Sta. Circ. 47 No. 3 (2012), 676-685.

[29] ABDUL-KARIM, M. A. H.-TAHA, S. M. R.-OMRAN, S. S. : Microprocessor-Based Implicit RMS Meter, International Journal of Electronics 62 No. 6 (1987), 953-959.

[30] MINAEI, S.-SAYIN, O. K.-KUNTMAN, H. : A New CMOS Electronically Tuneable Current Conveyor and its Application to Current-Mode Filters, IEEE Trans. Circuits Syst. I 53 No. 7 (2006), 1448-1457.

[31] GUM-Guide to the Expression of Uncertainty in Measurement, ISO 1993, 1993.

32] PROMMEE, P.-ANGKEAW, K.-SOMDUNYAKANOK, M. -DEJHAN, K.: CMOS-based Near Zero-Offset Multiple Inputs max-min Circuits and its Applications, Analog Integr. Circuits Signal Process. 61 (2009), 93-105.

33] ACHIGUI, H. J.-FAYOMI, C.-MAssiCotTE, D.-BOUKADOUM, M.: Low-Voltage, High-Speed CMOS Analog Latched Voltage Comparator using the Flipped Voltage Follower as Input Stage, Microelectronics Journal 42 (2011), 785-789.

[34] MAXIM Precision, CMOS Analog Switches, data sheet, 1994.

[35] Single Positive-Edge-Triggered D-type Flip-Flop SN74LVC1G80, Texsas Instruments, data sheet, 2007.

36] ANNEMA, A. J.-GOKSUN, G. A. : $0.0025 \mathrm{~mm}^{2}$ Bandgap Voltage Reference for 1.1V Supply in Standard $0.16 \mu \mathrm{m}$ CMOS, in Proceedings of 2012 IEEE International Solid-State Circuits Conference, 2012, pp. 364-366.

[37] BANBA, H.-SHIGA, H.-UMEZAWA, A.-MIYABA, T.TANZAWA, T.-ATSUMI, S.-AND SAKUI, K.: A CMOS bandgap Reference Circuit with sub-1V Operation, IEEE J. Solid State Circuits 34 No. 5 (1999), 670-674.

38] KAEWDANG, K.-KUMWACHARA, K.-SURAKAMPONTORN, W.: A Translinear-Based True RMS-to-DC Converter using only npn BJTs, AEU-Intern. Jour. Elec. Comm. 63 No. 6 (2009)), 472-477.

[39] YHANG, T.-EISENSTADT, W. R.-FOX, R. M.-YIN, Q. : Bipolar RMS Power Detectors, IEEE Jour. Sol. Sta. Circ. 41 No. 9 (2006), 2188-2192.

[40] SPENCER, R. R.: Analog Implementation of Artificial Neural Networks, in Proc. IEEE Int. Symp. Circuits Syst., vol. 2, 1991, pp. 1271-1274.

Received 7 March 2014

Predrag B. Petrović was born in Čačak, Yugoslavia, on January 26, 1967. He received the BSEE and MSc degrees in electrical engineering from the University of Belgrade Yugoslavia, in 1991 and 1994, respectively, and $\mathrm{PhD}$ degree in the field of digital signal processing at the University of Novi Sad in 2004. He finished faculty as one of the best (top 2\%) in his class. Since 1991, he has been the Teaching and Research Assistant at the University of Kragujevac, Cačak College of Engineering, and from 2011 he holds position of full professor. His main interest is digital signal processing, microcontroller programming, power electronics, AD conversion, mathematics, and cryptology. He published more then 150 journals and conference papers, five university books, one international monograph and holds five patents. He is the member of IEEE, IEICE and MENSA. 